\title{
A facile method for the selective decoration of graphene defects based on a galvanic displacement reaction
}

\author{
Juree Hong ${ }^{1}$, Jae-Bok Lee ${ }^{2}$, Sanggeun Lee ${ }^{1}$, Jungmok Seo ${ }^{1}$, Hyunsoo Lee ${ }^{3,4}$, Jeong Young Park ${ }^{3,4}$, \\ Jong-Hyun $\mathrm{Ahn}^{2}$, Tae Il Seo ${ }^{5}$, Taeyoon Lee ${ }^{1}$ and Han-Bo-Ram Lee ${ }^{6}$
}

Inherent defects, such as grain boundaries (GBs), wrinkles and structural cracks, present on chemical vapor deposition (CVD)-grown graphene are inevitable because of the mechanism used for its synthesis. Because graphene defects are detrimental to electrical transport properties and degrade the performance of graphene-based devices, a defect-healing process is required. We report a simple and effective approach for enhancing the electrical properties of graphene by selective graphene-defect decoration with Pd nanoparticles (Pd NPs) using a wet-chemistry-based galvanic displacement reaction. According to the selective nucleation and growth behaviors of Pd NPs on graphene, several types of defects, such as GBs, wrinkles, graphene regions on $\mathrm{Cu}$ fatigue cracks and external edges of multiple graphene layers, were precisely confirmed via spherical aberration correction scanning transmission electron microscopy, field-emission scanning electron microscopy and atomic force microscopy imaging. The resultant Pd-NP-decorated graphene films showed improved sheet resistance. A transparent heater was fabricated using Pd-decorated graphene films and exhibited better heating performance than a heater fabricated using pristine graphene. This simple and novel approach promises the selective decoration of defects in CVD-grown graphene and further exploits the visualization of diverse defects on a graphene surface, which can be a versatile method for improving the properties of graphene. NPG Asia Materials (2016) 8, e262; doi:10.1038/am.2016.42; published online 15 April 2016

\section{INTRODUCTION}

Chemical vapor deposition (CVD) has been successfully exploited as a promising method for fabricating graphene for diverse applications in different fields, including high-frequency electronics, ${ }^{1}$ energy devices ${ }^{2}$ and optoelectronics. ${ }^{3}$ Specifically, the most important advantage of CVD is the industrial-scale fabrication of graphene, which is essential for the commercialization of graphene technologies. Although the CVD method is more advantageous for the large-scale fabrication of graphene than other methods, it unavoidably introduces structural defects $^{4}$ such as vacancies, ${ }^{5}$ grain boundaries (GBs), ${ }^{6-8}$ wrinkles ${ }^{4,9,10}$ and cracks, ${ }^{11}$ which result in the deterioration of the physical and electrical properties of graphene. For instance, one-dimensional line defects of GBs and wrinkles are observed on CVD-grown single-layer graphene (SLG) because of its polycrystalline nature and because of the mismatch between the thermal expansion coefficients of graphene and the catalytic metal substrate during the cooling process of the CVD method, respectively. ${ }^{4,6,7,9}$ The presence of one-dimensional line defects is detrimental to graphene-based electronic devices. For example, the sheet resistance of graphene synthesized by CVD is approximately $1 \mathrm{k} \Omega \mathrm{sq}^{-1}$, which is much lower than the theoretical value. Recently, several groups have reported that the electronic transport properties can be improved by increasing the grain size of graphene via a micrometer-scale growth technique, ${ }^{12,13}$ bridging graphene grains using metal nanowires ${ }^{14}$ and selectively healing atomic defects in CVD-grown graphene through atomic layer deposition. ${ }^{15-17}$ However, despite improved electronic transport properties, these approaches generally require sophisticated treatments, high-cost equipment and a long processing time, which can be disadvantageous for mass production in terms of cost and simplicity. Recently, Ho et al. ${ }^{18}$ demonstrated a simple and cost-effective method to enhance the electrical properties of CVD-grown graphene by selectively healing the topological cracks in graphene with metallic nanoparticles using a solution-based reduction-oxidation reaction. Despite the simplicity of this method,

\footnotetext{
${ }^{1}$ Nanobio Device Laboratory, School of Electrical and Electronic Engineering, Yonsei University, Seoul, Korea; ${ }^{2}$ Flexible Electronics Laboratory, School of Electrical and Electronic Engineering, Yonsei University, Seoul, Korea; ${ }^{3}$ Center for Nanomaterials and Chemical Reactions, Institute for Basic Science (IBS), Daejeon, Korea; ${ }^{4}$ Graduate School of EEWS, Korea Advanced Institute of Science and Technology (KAIST), Daejeon, Korea; ${ }^{5}$ Department of Mechanical Engineering and Robotics, Incheon National University, Incheon, Korea and ${ }^{6}$ Department of Materials Science and Engineering, Incheon National University, Incheon, Korea

Correspondence: Professor T Lee, Nanobio Device Laboratory, School of Electrical and Electronic Engineering, Yonsei University, Room C312, Engineering Hall 3, 50 Yonsei-ro, Seodaemun-gu, Seoul 03722, Korea.

E-mail: taeyoon.lee@yonsei.ac.kr

or Professor H-B-R Lee, Department of Materials Science and Engineering, Incheon National University, Room 305, Building 8, 119 Academy-ro, Yeonsu-gu, Incheon 22012, Korea.

E-mail: hbrlee@inu.ac.kr

Received 30 October 2015; revised 11 February 2016; accepted 16 February 2016
} 
it did not heal inherent defects of graphene, such as GBs and wrinkles.

In this study, we demonstrate a facile and effective approach for the selective decoration of diverse types of inherent defects present in CVD-grown SLG with palladium nanoparticles (Pd NPs) via a wet-chemistry-based galvanic displacement reaction to enhance the electrical properties of graphene films. The wet-chemistry-based galvanic displacement reaction allows metal NPs to predominately nucleate on the intrinsic defects of graphene, such as wrinkles, GBs, cracks and external edges, because of the greater chemical reactivity at these defect sites. The selective deposition of Pd NPs on each defect site was directly confirmed by spherical aberration correction scanning transmission electron microscopy $\left(\mathrm{C}_{\mathrm{s}}\right.$-corrected STEM), field emission scanning electron microscopy (FE-SEM) and friction force microscopy. To confirm the improvement in electrical properties, we measured the changes in sheet resistance after the galvanic displacement reaction under the optimized reaction conditions. We obtained an improved electrical sheet resistance of $173 \Omega$ sq. ${ }^{-1}$ at $91.3 \%$ optical transmittance within a few minutes, which was comparable or superior to those of previously reported graphene-based transparent conducting electrodes. ${ }^{15}$ To explore the potential of defect-healed graphene as a transparent electrode, we fabricated a transparent heater using Pd-decorated graphene and compared the heating performance to that of a heater fabricated using pristine graphene. Notably, the Pd-decorated graphene exhibited better heating performance than pristine graphene because of its improved electrical sheet resistance. The results of the present study indicate that the decoration of graphene defects possibly contributes to a considerable improvement in the electrical properties of graphene. We believe that graphene containing defects healed with metal NPs, as prepared via our simple and effective galvanic displacement reaction method, can be potentially used in a broad range of applications, including automobile smart windows, smart mirrors, defogging/deicing systems and smart displays.

\section{MATERIALS AND METHODS}

\section{Graphene growth using the CVD process}

A $25-\mu \mathrm{m}$-thick $\mathrm{Cu}$ foil (Alfa Aesar) was loaded into a quartz tube furnace for the growth of SLG. Cu foil samples were annealed at $100{ }^{\circ} \mathrm{C}$ at low pressure for $2 \mathrm{~h}$ and $40 \mathrm{~min}$ under flowing (10 s.c.cm) hydrogen $\left(\mathrm{H}_{2}\right)$; the SLG was then formed by injecting 1 s.c.cm of methane $\left(\mathrm{CH}_{4}\right)$ as a carbon source for $5 \mathrm{~h}$ and $30 \mathrm{~min}$. After the growth of the SLG, the furnace was rapidly cooled to room temperature in the presence of $\mathrm{H}_{2}$. For the growth of incomplete SLG on $\mathrm{Cu}$ foil, the $\mathrm{CH}_{4}$ flow rate was decreased and the $\mathrm{H}_{2}$ annealing time was controlled. ${ }^{19}$

\section{Metal NP deposition process \\ Before the deposition of Pd NPs onto the graphene surface, the graphene on the backside of the $\mathrm{Cu}$ foil was removed using reactive ion etching with oxygen $\left(\mathrm{O}_{2}\right)$ plasma (70 mTorr, $100 \mathrm{~W}, 2 \mathrm{~s}$ ). The Pd NPs were then deposited onto the graphene surface by immersing the SLG/Cu foil sample in an aqueous $\mathrm{PdCl}_{2}$ solution. ${ }^{20}$ The concentration of the $\mathrm{PdCl}_{2}$ solution ranged from 0.01 to $5 \mathrm{~mm}$. The deposition mechanism of Pd NPs on graphene was based on the graphene- buffered galvanic displacement reaction between the $\mathrm{Cu}$ foil underneath the SLG layer and the metal-ion-containing salt solution. After the deposition of Pd $\mathrm{NPs}, \mathrm{SLG} / \mathrm{Cu}$ foil samples were rinsed with deionized water, dried with $\mathrm{N}_{2}$ and baked on a hot plate at $60^{\circ} \mathrm{C}$ for $10 \mathrm{~min}$ to evaporate the remaining water molecules.}

\section{Transfer of metal NPs/graphene onto an $\mathrm{SiO}_{2} / \mathrm{Si}$ substrate, polyethylene terephthalate, glass and a TEM grid}

As-prepared metal NPs/graphene/Cu foil samples were coated with poly(methyl methacrylate) (PMMA; Sigma Aldrich; average molar weight of approximately
996000 , dissolved in anisole to a concentration of $40 \mathrm{mg} \mathrm{ml}^{-1}$ ) using a spin-coater at 4000 r.p.m. for $30 \mathrm{~s}$. The PMMA-coated metal NPs/graphene samples were then floated on a $0.1-\mathrm{M}$ ammonium persulfate solution to etch the $\mathrm{Cu}$ foil. After the $\mathrm{Cu}$ foil was etched, the metal NPs/graphene samples with the PMMA support were moved to deionized water and incubated for $30 \mathrm{~min}$ in a floating state to rinse the remaining etchant residue. The metal NPs/graphene samples with the PMMA support were then manually transferred to the desired substrate $\left(\mathrm{SiO}_{2} / \mathrm{Si}\right.$ substrate, polyethylene terephthalate (PET), or glass) and then baked on a hot plate at $60^{\circ} \mathrm{C}$ for $30 \mathrm{~min}$. The PMMA support was then removed with acetone and additional thermal annealing. When preparing the metal NPs/graphene samples on a perforated silicon nitride TEM grid, we used a direct transfer method. ${ }^{21}$ The TEM grid was placed on top of the graphene on $\mathrm{Cu}$, and then, a drop of isopropyl alcohol was gently dropped onto the TEM grid. The evaporation of isopropyl alcohol induced strong contact between the graphene and TEM grid. The TEM-grid-contacting metal NPs/graphene samples were floated on a $0.1-\mathrm{M}$ ammonium persulfate solution to etch the $\mathrm{Cu}$ foil, floated on deionized water to rinse the remaining residues and then dried at ambient temperature and pressure.

\section{Defect-heated graphene-based heaters}

A 100-nm-thick Cu electrode was deposited onto a PET substrate via a thermal evaporation system. To transfer the as-prepared $\mathrm{Pd}$-decorated graphene $(20 \mathrm{~mm} \times 20 \mathrm{~mm})$ onto the electrode-patterned PET substrate, a PMMA support was spin-coated. To etch out the $\mathrm{Cu}$ foil, the PMMA-coated Pd $\mathrm{NP} /$ graphene/ $\mathrm{Cu}$ stack was floated on a 0.1-M ammonium persulfate solution. After etching of the $\mathrm{Cu}$ foil, the floating PMMA-coated Pd NP/graphene stack was floated on deionized water to remove any remaining etchant residue. The floating PMMA-coated Pd NP/graphene stack was then manually transferred to a PET substrate with pre-defined electrodes, which was subsequently baked on a hot plate at $60^{\circ} \mathrm{C}$ for $30 \mathrm{~min}$. Direct current power was applied by a power supply (Keithley 2400) to the heater through the $\mathrm{Cu}$ electrodes. The timedependent temperature profiles were observed using an infrared thermal imaging camera (FLIR t630).

\section{Characterization techniques}

The morphologies of the metal NPs on graphene were characterized using FE-SEM (JEOL JSM-7001F), AFM (JPK Instrument Nanowizard) and $\mathrm{C}_{\mathrm{s}}$-corrected STEM (JEM-ARM 200F). The surface coverage of Pd on SLG was calculated from an area analysis of several FE-SEM images. To clearly visualize the morphology of the SLG surface in plan-view FE-SEM images, we performed the transfer process for Pd NPs/SLG on $\mathrm{SiO}_{2} / \mathrm{Si}$ substrates after the galvanic displacement reaction in a $0.01-\mathrm{mM}^{\mathrm{PdCl}_{2}}$ solution. ${ }^{19}$ The $\mathrm{C}_{\mathrm{s}}$-corrected STEM was operated at an accelerating voltage of $80 \mathrm{kV}$. The elemental composition of the metal NPs on SLG after the galvanic displacement reaction was investigated using the FE-SEM energy-dispersive spectrometry link system (JEOL 3000F). Raman spectroscopy and spatial Raman mapping were performed using a micro-Raman system (Jobin-Yvon, LabRam HR) equipped with a motorized sample stage to monitor the deformation of graphene. The wavelength of the excitation laser was $532 \mathrm{~nm}$, its power was set to $5 \mathrm{~mW}$ and its spot size was approximately $1 \mu \mathrm{m}$. The friction force microscopy and non-contact AFM measurements were performed under ambient conditions (Agilent $5500 \mathrm{AFM}$ ). ${ }^{22}$ The sheet resistances of metal NPs/graphene on PET and glass substrates were determined using a standard four-probe method. The transmittance of metal NPs/graphene on PET and glass substrates in the spectral range from 400 to $800 \mathrm{~nm}$ was measured using a UV/visible spectrophotometer (JASCO V-650).

\section{RESULTS AND DISCUSSION}

Previous studies on graphene-buffered galvanic displacement reactions have shown either uniform metal film deposition or randomly distributed metal nanoparticle deposition onto graphene surfaces. ${ }^{20}$ Figure 1a shows a continuous Pd film on a CVD-grown SLG surface after a galvanic displacement reaction in a 5-mm palladium chloride $\left(\mathrm{PdCl}_{2}\right)$ solution for $5 \mathrm{~min}$, which is consistent with the results of a previous report. ${ }^{20}$ However, when the concentration of the $\mathrm{PdCl}_{2}$ 
a

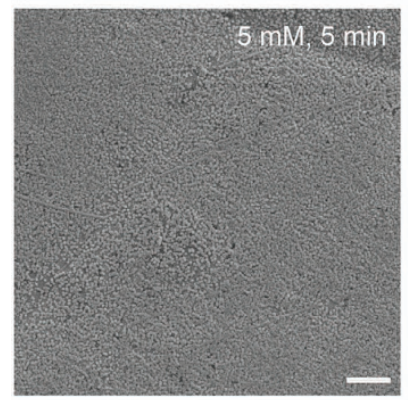

d

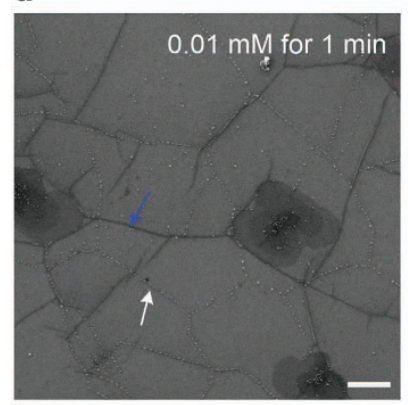

g

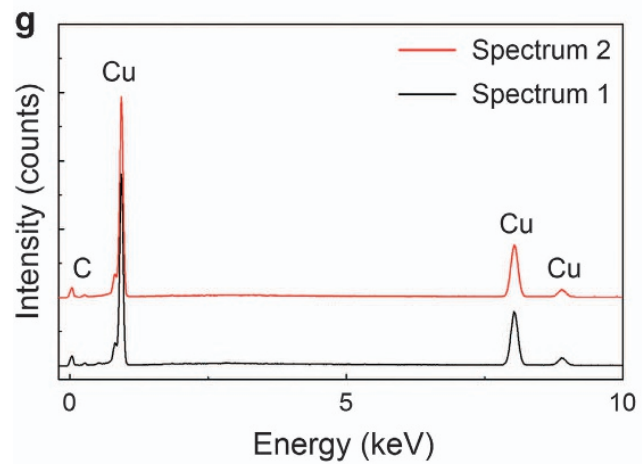

b

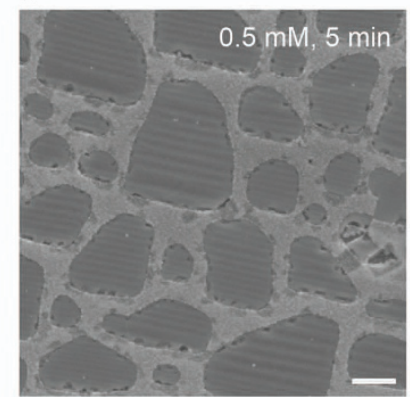

e

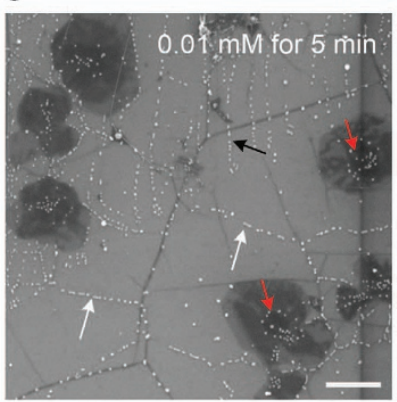

C

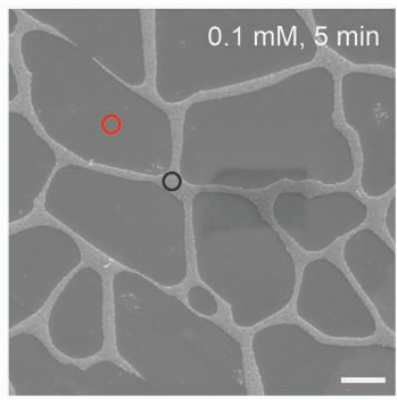

f

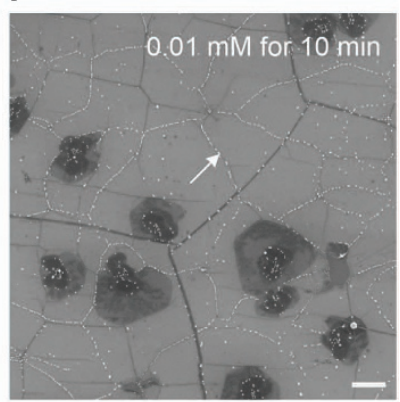

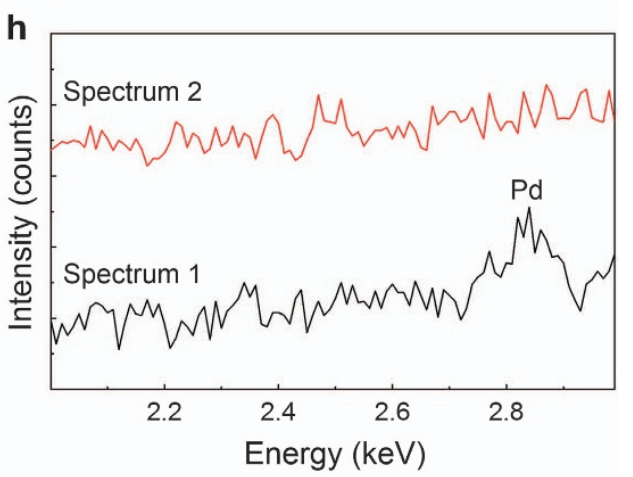

Figure 1 Plan-view FE-SEM micrographs of Pd-deposited SLG/Cu foil samples after galvanic displacement reaction for $5 \mathrm{~min}$ in a $\mathrm{PdCl}{ }_{2}$ solution with a concentration of (a) $5 \mathrm{~mm}$, (b) $0.5 \mathrm{~mm}$ or (c) $0.1 \mathrm{~mm}$. Scale bars indicate $1 \mu \mathrm{m}$. Plan-view FE-SEM micrographs of Pd NP-deposited SLG/SiO $2 / \mathrm{Si}$ samples after galvanic displacement reaction in a $0.01-\mathrm{mm} \mathrm{PdCl}_{2}$ solution for (d) $1 \mathrm{~min}$, (e) $10 \mathrm{~min}$ and (f) $30 \mathrm{~min}$. All scale bars indicate $500 \mathrm{~nm}$. (g and $\mathbf{h}$ ) energydispersive spectrometry spectra collected at two different regions (spectrum 1 from the black circle and spectrum 2 from the red circle in c). FE-SEM, field emission scanning electron microscopy; NP, nanoparticle; SLG, single-layer graphene.

solution was decreased to $0.5 \mathrm{~mm}$, irregular polygonal structures were formed, as shown in Figure 1b, which has not been previously reported in investigations using the same method. When the concentration of the $\mathrm{PdCl}_{2}$ solution was further decreased to $0.1 \mathrm{~mm}$, both the width and overall surface coverage of the irregular polygonal structure decreased, as shown in Figure 1c. Energydispersive spectrometry was performed at two points (the black and red circles in Figure 1c). As shown in Figures $1 \mathrm{~g}$ and h, spectrum 1, which was collected from the black-circled area, shows a Pd energydispersive spectrometry peak at $2.84 \mathrm{keV}$, whereas spectrum 2, which was collected from the red-circled area, shows no Pd energy-dispersive spectrometry peak, indicating that the irregular polygonal structure is composed of Pd. The selective deposition of Pd onto the SLG surface was only observed when the concentration of the $\mathrm{PdCl}_{2}$ solution was less than $0.5 \mathrm{~mm}$. The surface coverage of Pd NPs increased with increasing concentration of $\mathrm{PdCl}_{2}$. We confirmed that irregular polygonal structures were sparsely filled with Pd NPs when the concentration of the $\mathrm{PdCl}_{2}$ solution was less than $0.02 \mathrm{~mm}$.
By contrast, the irregular polygonal structures were completely filled with Pd NPs deposited from the 0.1- $\mathrm{mm} \mathrm{PdCl}_{2}$ solution for more than $1 \mathrm{~min}$, resulting in nanowire-like $\mathrm{Pd}$ structures (Supplementary Figure S1). When the concentration of the $\mathrm{PdCl}_{2}$ solution exceeded $0.5 \mathrm{~mm}$, Pd NPs were deposited onto the entire SLG surface, but the deposition was not selective.

We intentionally decreased the concentration of the $\mathrm{PdCl}_{2}$ solution to $0.01 \mathrm{~mm}$ to investigate the change of Pd morphology on SLG during the initial growth stage with increasing galvanic displacement reaction time. To clearly observe only the change in Pd without interference from the $\mathrm{Cu}$ foil roughness in the SEM analysis, we transferred the Pd-deposited SLG to an $\mathrm{SiO}_{2}$ substrate. We confirmed that the Pd NPs remained intact even after the transfer process (Supplementary Figure S2). Figures $1 \mathrm{~d}-\mathrm{f}$ show the representative plan-view FE-SEM images of Pd-deposited SLG layers as a function of the galvanic displacement reaction time. Pd NPs sparsely filled the edges of the irregular polygonal structures (white arrow in Figure 1d) after $1 \mathrm{~min}$ of galvanic displacement reaction in the $0.01-\mathrm{mM}^{\mathrm{PdCl}_{2}}$ solution. 
The dark-contrast region in Figure 1d was presumed to be multiple graphene layers grown during the CVD process. ${ }^{19}$ Alternatively, the line-forming region marked with a blue arrow in Figure 1d indicates that extra wrinkles were generated during the transfer process. ${ }^{23}$ When the galvanic displacement reaction time was increased to $5 \mathrm{~min}$, the size and number of Pd NPs increased, as shown in Figure 1e. Interestingly, Pd NPs were formed along the edges of the polygonal structures (white arrows) and in parallel lines (black arrow). In addition, Pd NPs irregularly formed around the dark regions denoted by the red arrows. When the galvanic displacement reaction time was further increased to $10 \mathrm{~min}$, the amount of Pd NPs increased along the edges of the polygonal structures such that the polygonal structures are recognizable in Figure 1f. In addition, Pd NPs on the parallel lines and polygonal structures were also observed after a 10-min reaction, and the Pd NPs that formed on the edges of the polygonal structures were nearly connected after reacting for $30 \mathrm{~min}$ (see Supplementary Figures S1a and S1b). At a fixed concentration, the amount of Pd NPs increased with increasing reaction time, and the Pd NPs were selectively formed on three different structures: the edges of polygonal structures, parallel structures and multiple graphene layers. The different formations of Pd NPs will be discussed in conjunction with various $\mathrm{C}_{\mathrm{s}}$-corrected STEM, FE-SEM and friction force microscopy analyses in the following sections.

The Pd NPs that formed along two types of lines, denoted by the white and black arrows in Figure 1e, were analyzed using $\mathrm{C}_{\mathrm{s}}$-corrected STEM. Figure 2a shows a $\mathrm{C}_{\mathrm{s}}$-corrected STEM image captured in the region where the Pd NPs formed on the polygonal lines. Three different areas separated by the Pd NP lines are shown at high magnification in Figures $2 \mathrm{~b}-\mathrm{d}$. The average size of the Pd NPs was approximately $12 \mathrm{~nm}$ and most of the Pd NPs were interconnected. Selective-area diffraction patterns (SADPs) were acquired from three regions denoted by the colored boxes in Figure 2a. Each SADP clearly shows a hexagonal point pattern in Figures $2 \mathrm{e}-\mathrm{g}$ because of the hexagonal crystal structure of graphene. ${ }^{8}$ However, the orientation of each SADP was unique, and the relative misorientation angles were calculated with respect to the lattice orientation of one specific region. Because the orientation of the SADP reflects the original crystal structure, the different orientations indicate that the three areas divided by the Pd NP lines have different lattice orientations. ${ }^{6}$ Therefore, the Pd NPs were deposited along the GBs of the graphene. The parallel lines were also analyzed using SADP, as shown in Figure $2 \mathrm{~h}$. The SADPs collected from area 4 and area 5 show the same orientation as shown in Figures $2 \mathrm{i}$ and $\mathrm{j}$. This result indicates that parallel Pd NPs were not deposited onto GBs of graphene but on different surface features that more easily initiate nucleation of Pd NPs than other pristine regions. Many surface features supportive of the formation of parallel lines can be generated during graphene synthesis and transfer processes, such as graphene wrinkles, overlapped graphene layers and shallow parallel lines on $\mathrm{Cu}$ foil. Because graphene wrinkles and overlapped graphene layers have various shapes depending on their growth conditions, ${ }^{9,24}$ they cannot form regular parallel lines, as observed in our results. Shallow parallel lines on $\mathrm{Cu}$ foil, which are called fatigue cracks, originate from the mechanical processing of the $\mathrm{Cu}$ foil. ${ }^{24} \mathrm{Cu}$ fatigue cracks have a very specific type of shallow lines with a spacing of approximately $200 \mathrm{~nm}$. In addition, the topographical features of the underlying $\mathrm{Cu}$ foil, such as fatigue cracks, can be transferred to synthesized graphene by $\mathrm{CVD}^{23,25}$ such that the shallow lines on the $\mathrm{Cu}$ foil can cause local fractures on the graphene. In our results, the line shape and spacing of the Pd parallel lines are nearly identical to the shallow lines of the $\mathrm{Cu}$ fatigue cracks.
Therefore, the parallel lines of Pd NPs are deposited onto the shallow lines.

During the galvanic displacement reaction, the deposition of $\mathrm{Pd}$ NPs occurs through a simple spontaneous redox reaction between the $\mathrm{Cu}$ foil beneath the SLG and $\mathrm{Pd}^{2+}$ ions in the $\mathrm{PdCl}_{2}$ aqueous solution. The SLG acts as a cathode by delivering electrons for Pd deposition, whereas the $\mathrm{Cu}$ foil serves as an anode at which $\mathrm{Cu}$ atoms are ionized into $\mathrm{Cu}^{2+}$ and then dissolved. Defect sites present on the SLG layer, including GBs and intrinsic topological defects, act as electronically favorable sites for $\mathrm{Pd}$ deposition because of their chemical instability. ${ }^{25,26}$ Therefore, graphene imperfections effectively function as nucleation sites, as observed when Pd NPs were formed on both graphene GBs and graphene regions over $\mathrm{Cu}$ fatigue cracks. Defect sites that act as nucleation sites for Pd can be generated during the galvanic displacement reaction. Figure $2 \mathrm{k}$ shows the micro-Raman spectra of two different samples, a pristine graphene sample (denoted by the black solid line) and an SLG/Cu sample after galvanic reaction with a $0.01-\mathrm{mm} \mathrm{PdCl}_{2}$ solution for $10 \mathrm{~min}$ (denoted by the red solid line). Only minor changes were observed in the $\mathrm{D}, \mathrm{G}$ and $2 \mathrm{D}$ peak intensities in the Raman spectrum, indicating that little damage occurred to the graphene structure. Alternatively, positional blueshifts in both the $\mathrm{G}$ and $2 \mathrm{D}$ bands were observed, which implies a doping effect of graphene, likely because of the formation of Pd NPs on SLG. ${ }^{27,28}$ The doping effect will be discussed along with the dependence of the sheet resistance results on the Pd surface coverage in the following section. Inherent defects on graphene function as reactive sites for the deposition of Pd during the galvanic displacement reaction, and the galvanic displacement reaction has no influence on the additional generation of defects.

The graphene GBs and shallow lines from the $\mathrm{Cu}$ fatigue cracks are favorable sites for Pd nucleation during the galvanic displacement reaction. Thus, graphene wrinkles might also act as reactive sites for the deposition of Pd NPs because graphene wrinkles are a locally fractured region on the graphene surface, which results in an increase in chemical reactivity. ${ }^{23}$ First-principles calculations in other studies have demonstrated that graphene wrinkles have higher binding energy than GBs. ${ }^{25}$ Because graphene wrinkles are produced as a consequence of the different thermal expansion coefficients of graphene and $\mathrm{Cu}$ during the CVD growth process, ${ }^{4,9,24,29}$ wrinkle shapes, which are indistinguishable from other various structures, vary depending on the growth conditions. Therefore, we prepared pristine graphene, which has numerous wrinkles, by controlling the CVD growth conditions to investigate Pd deposition onto graphene wrinkles during the galvanic displacement reaction. Figures $3 a-c$ show plan-view FE-SEM images before and after the galvanic displacement reaction of the wrinkled graphene samples. The white arrow in Figure $3 \mathrm{a}$ indicates wrinkles on graphene that were intentionally generated by controlling the growth conditions. After the galvanic displacement reaction, these wrinkled regions were covered with Pd NPs, as shown in Figures $3 \mathrm{~b}$ and c. When the galvanic displacement reaction was performed in the 0.01-mM $\mathrm{PdCl}_{2}$ solution for $5 \mathrm{~min}$, polygonal structures were similarly observed; however, double Pd lines formed along the edges of the polygonal structures. Wrinkles have higher chemical reactivity than pristine regions because of their enhanced $s p^{3}$ characteristic arising from high local curvatures. ${ }^{30}$ Therefore, Pd NPs are deposited along wrinkles with polygonal shapes, leading to the formation of polygonal Pd lines. In addition, if the height of a wrinkle is sufficient, the wrinkle collapses, leading to the formation of two curved sides (see Supplementary Figure S3). Therefore, double Pd lines are formed along the two curved sides because of the high chemical reactivity of the high curvature. ${ }^{25}$ When the concentration of the $\mathrm{PdCl}_{2}$ solution 

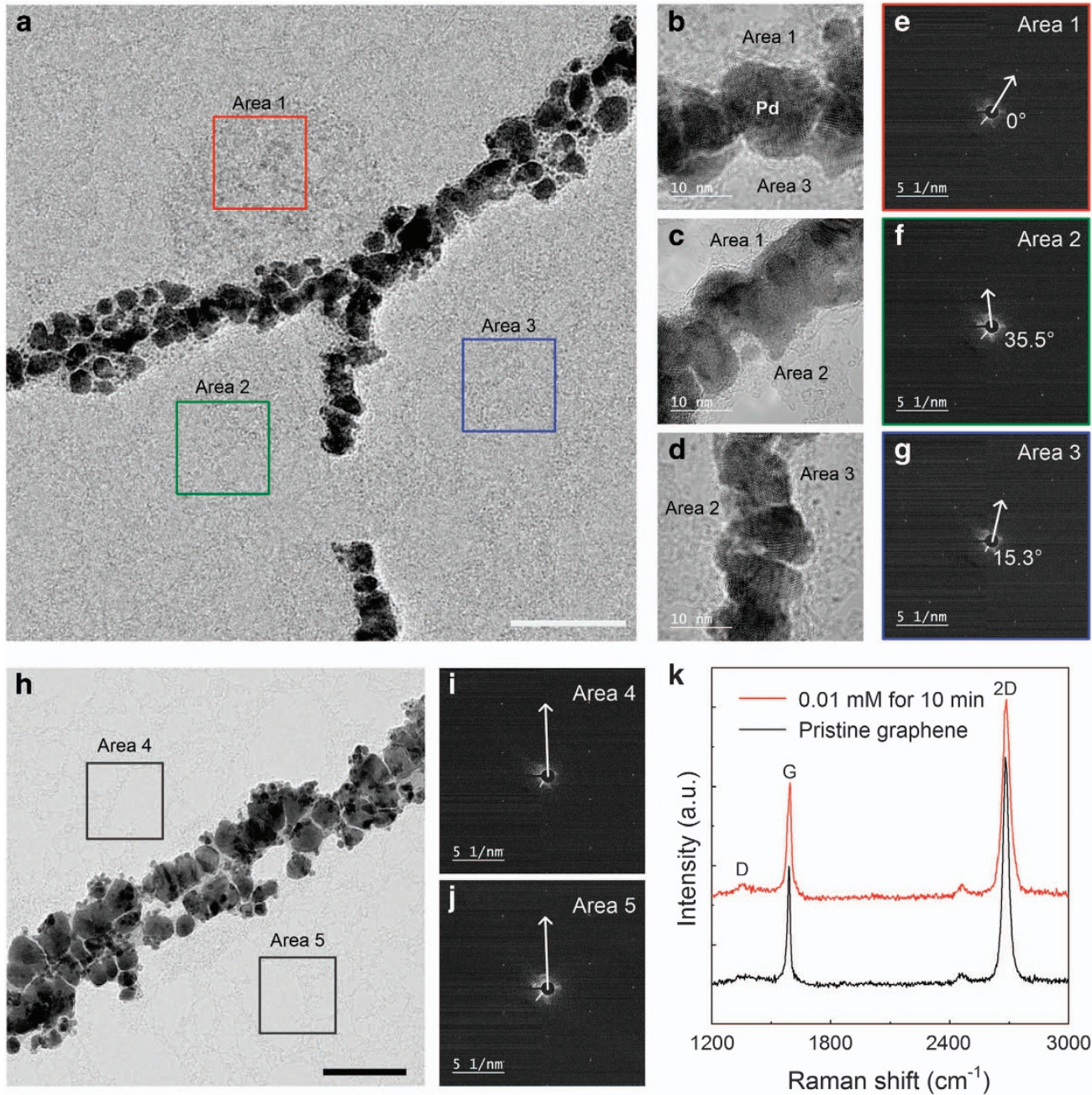

Figure 2 (a) A $\mathrm{C}_{\mathrm{s}}$-corrected STEM image of a Pd-deposited SLG sample with irregular polygonal Pd structures after a galvanic displacement reaction in a 0.01-mM $\mathrm{PdCl}_{2}$ solution for $10 \mathrm{~min}$. Pd-deposited SLG was suspended on a silicon nitride grid. The scale bar indicates $50 \mathrm{~nm}$. (b-d) Atomic-resolution $\mathrm{C}_{\mathrm{s}}$-corrected STEM images of each area. The scale bars indicate $10 \mathrm{~nm}$. (e-g) SAED patterns of areas 1,2 and 3. The relative misorientation angle of graphene grains with respect to area 1 is shown for each area. The scale bar indicates $5 \mathrm{~nm}^{-1}$. (h) A $\mathrm{C}_{\mathrm{s}}$-corrected STEM image of Pd-deposited SLG with parallel Pd structures. (i and j) SAED patterns of area 4 and area 5 . The scale bars indicate $50 \mathrm{~nm}, 5 \mathrm{~nm}^{-1}$ and $5 \mathrm{~nm}^{-1}$, respectively. (k) Micro-Raman spectra of pristine graphene (black solid line) and $\mathrm{Pd}$-deposited $\mathrm{SLG}$ on an $\mathrm{SiO}_{2} / \mathrm{Si}$ substrate after the galvanic displacement reaction at a $\mathrm{PdCl}{ }_{2}$ solution concentration of $0.01 \mathrm{~mm}$ for $10 \mathrm{~min}$ (red solid line). SAED, selective-area electron diffraction; SLG, single-layer graphene; STEM, scanning transmission electron microscopy.

and the reaction time were increased to $0.1 \mathrm{~mm}$ and $10 \mathrm{~min}$, respectively, $\mathrm{Pd}$ was deposited on all edges of the polygonal structures.

Although the reasons for the formation of GBs and wrinkles differ, the shapes are similar to those of the polygonal structures, leading to the formation of Pd polygonal structures in both the cases. Thus, distinguishing whether Pd is formed on GBs or wrinkles is not easy and is a suggestion for future research. GB-free single-domain graphene was fabricated by controlling the growth conditions to demonstrate the formation of Pd on wrinkles. ${ }^{19}$ The graphene growth was interrupted immediately before the formation of a single polycrystalline graphene layer such that the GB-free single-domain graphene layers were not connected, as shown in Figure 3d. After the galvanic reaction in the $0.01-\mathrm{mM}^{\mathrm{PdCl}_{2}}$ solution for $10 \mathrm{~min}, \mathrm{Pd}$ lines were observed inside the single-domain graphene, as indicated with white arrows in Figure 3e, whereas a continuous Pd film was formed on the exposed $\mathrm{Cu}$ regions because of direct electron exchange between the $\mathrm{Cu}$ and $\mathrm{Pd}^{2+}$ ions, as shown in Figure $3 \mathrm{f}$. The formation of Pd lines inside the single-domain graphene clearly shows that the wrinkles acted as reactive sites for the nucleation of Pd NPs. Figure 3 shows that one-dimensional graphene wrinkles, the most abundant structural imperfections in graphene, can also be reactive sites for nucleation of Pd during the galvanic displacement reaction.

Figures $4 \mathrm{a}-\mathrm{c}$ show magnified plan-view FE-SEM images of Pd NPs deposited on multiple graphene layers after the galvanic displacement reaction, which is shown in Figures le and f. On the basis of the contrast difference, the number of graphene layers is considered to be 3 or 4 in Figures $4 \mathrm{a}-\mathrm{c}$. Because the edge of the graphene layer has dangling bonds, it is chemically active and can initiate Pd nucleation. In a previous report, the step edges of graphite were used as nucleation centers in atomic layer deposition (ALD) because of their high reactivity. ${ }^{15}$ Therefore, we expected that the edges of each graphene layer would be decorated by Pd NPs. However, Pd NPs were only formed on the edges of a specific graphene layer. To closely observe the selective deposition of Pd NPs, topography AFM and friction images of the Pd NP-deposited SLG sample were investigated on the basis of a previously reported analysis regarding the atomic friction on 

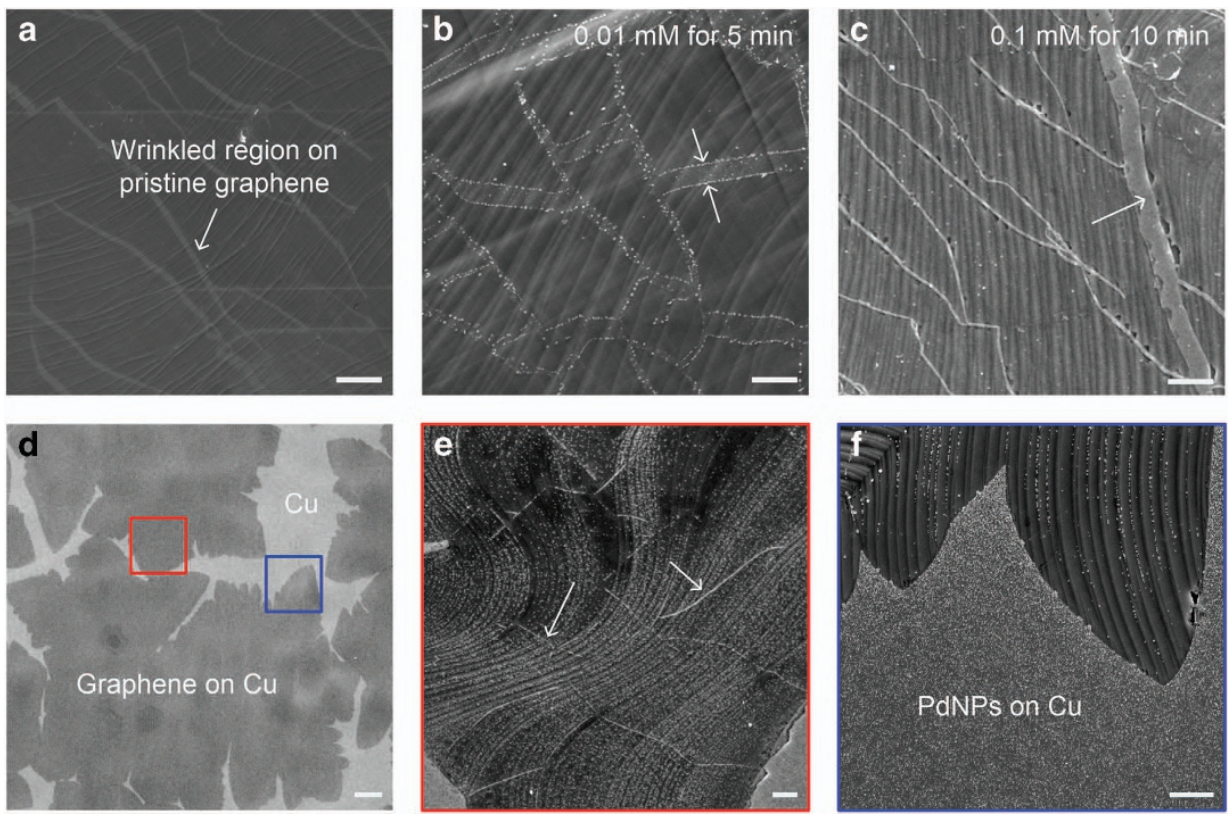

Figure 3 Plan-view FE-SEM micrographs of (a) pristine graphene, which has wrinkled regions, (b) Pd-deposited SLG on Cu foil after a galvanic displacement reaction in a 0.01-mM $\mathrm{PdCl}_{2}$ solution for $5 \mathrm{~min}$ and (c) Pd-deposited SLG on Cu foil after a galvanic displacement reaction in a $0.1-\mathrm{mm} P d C l_{2}$ solution for $10 \mathrm{~min}$. The scale bars indicate $1 \mu \mathrm{m}$. (d) A plan-view FE-SEM micrograph of the incomplete growth of SLG on Cu foil. The scale bar indicates $10 \mu \mathrm{m}$. Magnified plan-view FE-SEM micrographs of (e) the graphene region (red box in $\mathbf{d}$ ) and (f) Cu region (blue box in d). The scale bars indicate $1 \mu \mathrm{m}$. FE-SEM, field emission scanning electron microscopy; NP, nanoparticle; SLG, single-layer graphene.

the internal and external edges of graphite. ${ }^{22}$ In the topographical image shown in Figure $4 \mathrm{~d}$, the stacked graphene layers and edges are clearly observed such that the graphene layers can be labeled as (i), (ii), (iii) and (iv). On the basis of the topological image, we expected layer (i) to be placed at the bottom of the stack and layers (ii), (iii) and (iv) to be sequentially placed on top. However, in the friction image, only the edge of layer (iii) shows enhanced friction signals, as shown in Figure $4 \mathrm{e}$, Figure $4 \mathrm{~g}$ shows three line profiles in the topological image and frictional images. The profiles in the topological image along the $\mathrm{A}, \mathrm{B}$ and $\mathrm{C}$ lines clearly show consistent height differences, whereas the friction signals show the features of only the crossing edge of layer (iii). The friction differences are caused by the different chemical states between the internal (buried) and external (exposed) step edges at stacked graphene layers. Thus, the four graphene layers can have stacking configurations other than the order of (i), (ii), (iii) and (iv) expected on the basis of the topological image. The exact crossview configuration cannot be confirmed because of measurement limitations, resulting in four possible configurations for consideration (see Supplementary Figure S4). Although the stacking order cannot be confirmed, the friction force microscopy results show that layer (iii) has only external edges, as shown in the schematic of Figure $4 \mathrm{f}$. Layer (iii) has an external edge (denoted as a solid black line in the plan-view configuration shown in Figure 4f), whereas layers (ii) and (iv) have internal edges that are buried under layer (i) and layer (iii) (denoted as a dashed black line in the plan-view configuration in Figure 4f), respectively. Because the external edges of graphene show greater chemical reactivity than the basal plane of graphene, Pd NPs prefer to nucleate on the external edges of graphene. ${ }^{15,31}$ In contrast to the external edges, the internal edges do not initiate Pd nucleation because they are covered by another graphene layer. ${ }^{22}$ Therefore, the selective deposition of Pd NPs shown in Figures 4a-c occurred along the external edges of the graphene layer, such as layer (iii), but not the internal edges. In addition, densely concentrated Pd NPs in the center of multiple graphene layers, as shown in layer (iv), are attributed to defect sites of the nucleation center of the graphene. ${ }^{13}$

The stacking configuration of the multiple layers is an important evidence of the graphene nucleation and growth mechanism. Because of the self-limiting growth of graphene on $\mathrm{Cu}$ by CVD, the formation of multilayers is not clearly understood. After the analysis of the stacking configuration of the multiple layers using HR-TEM, ${ }^{32}$ several hypotheses, such as an adsorption-diffusion mechanism and a penetration mechanism of the carbon sources, have been proposed. ${ }^{33,34}$ However, few options exist for determining the configuration except HR-TEM, which requires a complicated sample preparation process and expensive tools. For the first time, a wet-chemistry-based galvanic displacement reaction was used to rapidly visualize the configurations of multiple graphene layers by tracing the external edges. This galvanic method can be utilized as a simple analysis method to investigate the nucleation and growth of $2 \mathrm{D}$ materials, including graphene.

The mechanism of selective Pd deposition onto different types of graphene defects is based on the graphene-buffered galvanic displacement reaction schematically illustrated in Figure 5. Theoretically, when a chemically and structurally inert SLG sample on $\mathrm{Cu}$ is immersed in a metal-ion-containing salt solution, metal NPs are uniformly deposited onto the basal plane of SLG through a simple spontaneous redox reaction between the $\mathrm{Cu}$ underneath SLG and the metal-ioncontaining salt solution. ${ }^{20,35}$ In this reaction, $\mathrm{Cu}$ acts as a reducing agent, whereas SLG functions as a buffer layer to transport electrons from the $\mathrm{Cu}$ for metal-ion nucleation. However, when chemical and structural defects are present on the SLG layer surface, the redox reaction between the $\mathrm{Cu}$ underneath the SLG and metal ions is substantially different. Because one-dimensional line defects and intrinsic topological defects on CVD-grown SLG are chemically reactive due to unstable bonding configurations, ${ }^{15,16,25,36}$ metal NPs are predominantly deposited onto these defect sites to compliment the 

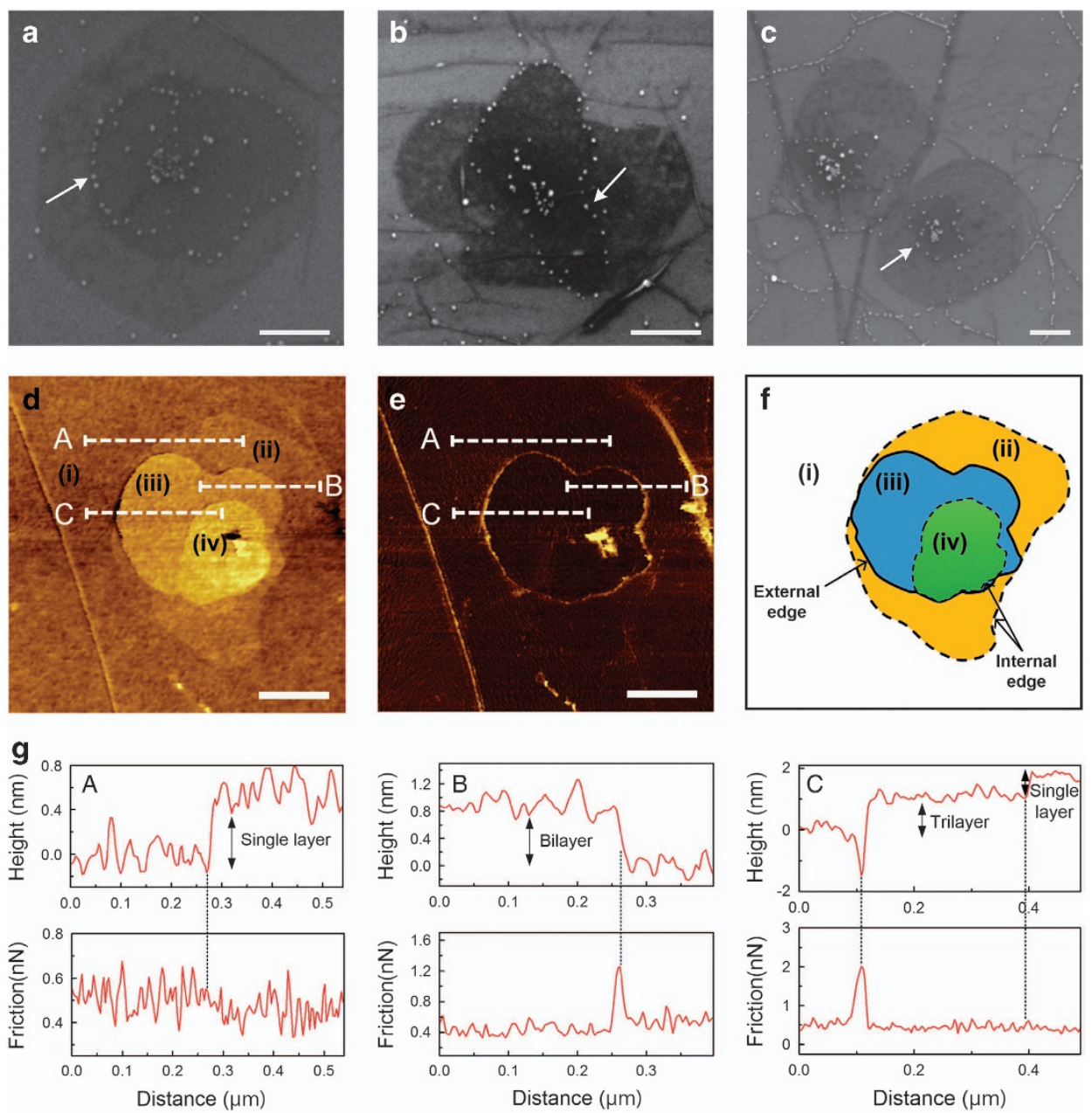

Figure 4 (a-c) Plan-view FE-SEM micrographs of Pd-NP-deposited SLG/SiO $/$ Si samples after a galvanic displacement reaction in a $0.01-\mathrm{mm} \mathrm{PdCl}_{2}$ solution for $10 \mathrm{~min}$. The white arrows indicate the formation of Pd NP lines. The scale bars indicate $250 \mathrm{~nm}$. (d) The $1.2 \mu \mathrm{m} \times 1.2 \mu \mathrm{m}$ topography and (e) friction images of Pd-NP-deposited graphene on an $\mathrm{SiO}_{2} / \mathrm{Si}$ substrate under an applied load of $15 \mathrm{nN}$ after a galvanic displacement reaction in a $0.01-\mathrm{mm} \mathrm{PdCl} 2$ solution for 10 min. (f) Schematic diagram showing external and internal edges of multiple grain domains (i) to (iv). Internal and external edges are indicated by dashed and solid black lines, respectively. (g) Line profiles of the height and friction along the dashed white lines denoted as A-C in d and e. FE-SEM, field emission scanning electron microscopy; NP, nanoparticle; SLG, single-layer graphene.

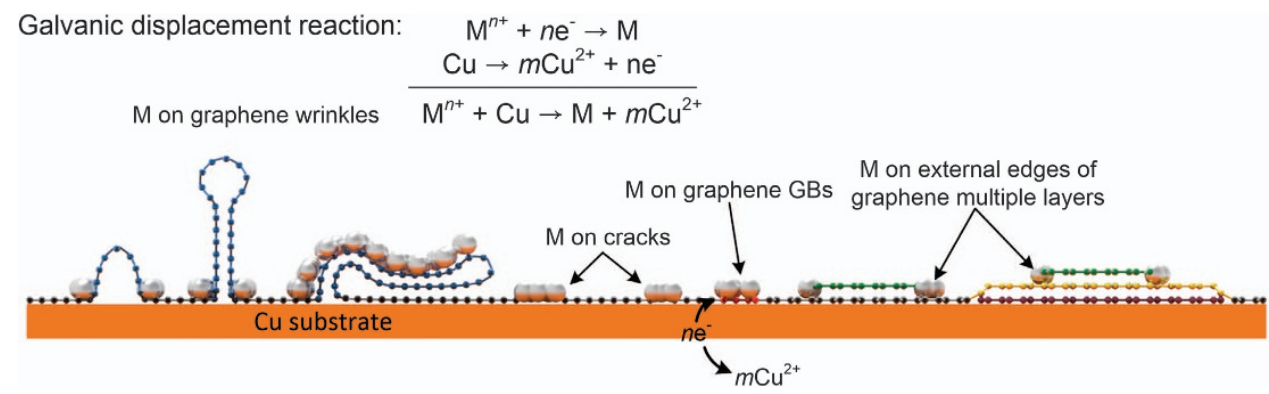

Figure 5 Schematic of the selective decoration process of CVD-grown SLG on Cu foil. The SLG shows diverse defects, such as wrinkles, cracks, grain boundaries and external edges. The decoration was performed via a wet-chemistry-based galvanic displacement reaction. CVD, chemical vapor deposition; SLG, single-layer graphene.

chemical instability. ${ }^{25}$ As noted in the experiments, selective $\mathrm{Pd}$ deposition was clearly observed after the galvanic displacement reaction at a $\mathrm{PdCl}_{2}$ concentration less than $0.5 \mathrm{~mm}$ because nucleation was initiated on the graphene defects. Alternatively, the galvanic displacement reaction at a concentration of $\mathrm{PdCl}_{2}$ greater than
$0.5 \mathrm{~mm}$ resulted in non-selective deposition of Pd onto SLG, consistent with previous reports. ${ }^{37}$ The random nucleation on graphene, irrespective of defect and pristine sites, likely requires a critical concentration to increase the impingement rate of ions and the reaction rate on the graphene surface. Consequently, the selective 

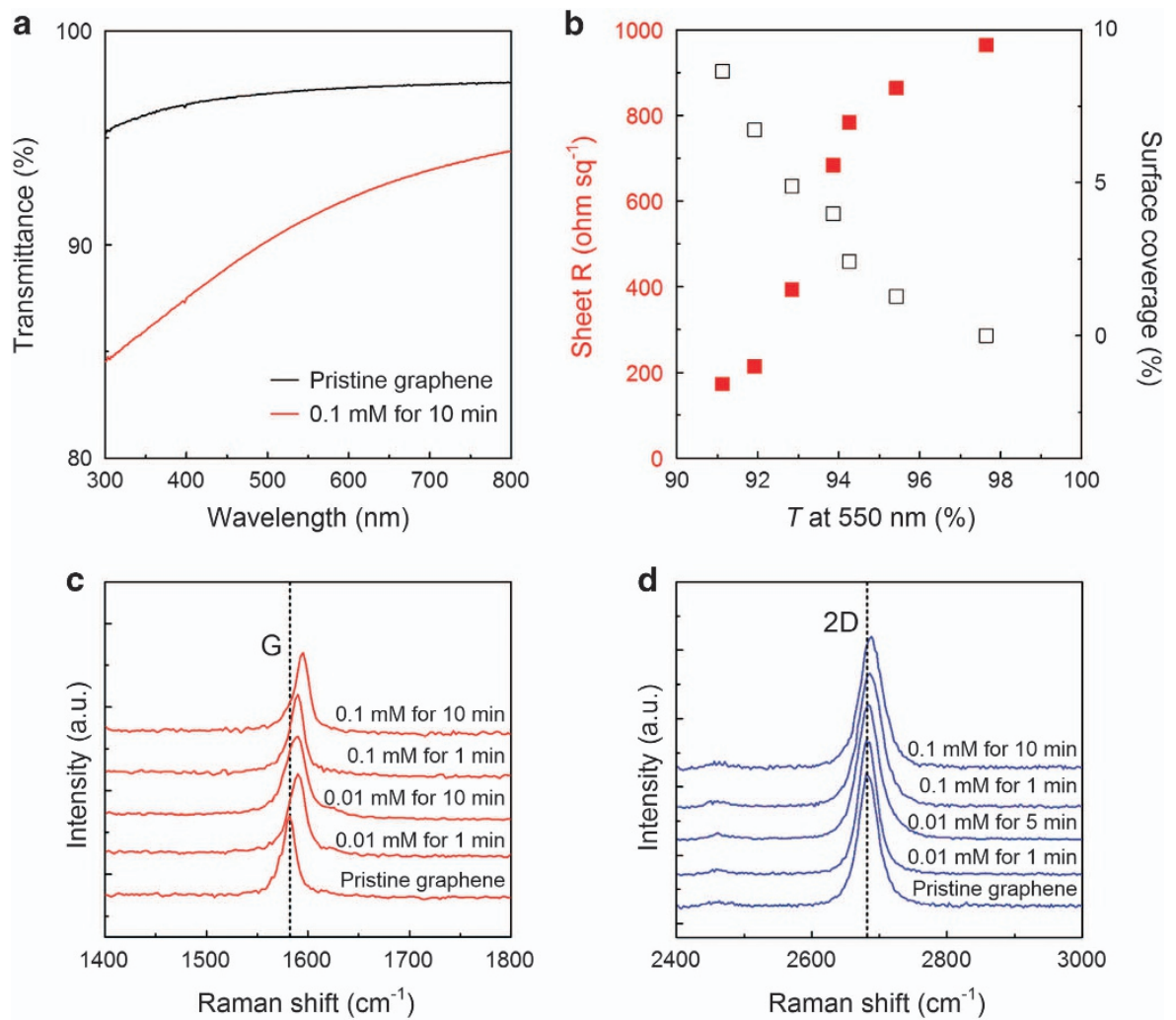

Figure 6 (a) Optical transmittance measurements of pristine graphene and a Pd-NP-deposited SLG sample reacted in a 0.01-mm PdCl 2 solution for 10 min. (b) Sheet resistance versus optical transmittance and surface coverage versus optical transmittance at $550 \mathrm{~nm}$ for various Pd depositions. (c) G-band Raman spectra of SLG on an $\mathrm{SiO}_{2} / \mathrm{Si}$ substrate for various Pd depositions and (d) 2D-band Raman spectra of $\mathrm{SLG}$ on an $\mathrm{SiO}_{2} / \mathrm{Si}$ substrate for various Pd depositions. NP, nanoparticle; SLG, single-layer graphene.

deposition of Pd onto the graphene surface can be controlled by varying both the solution concentration and the reaction time (see Supplementary Figure S5). In addition, because the wet-chemistrybased galvanic displacement reaction occurs as long as the redox potential of the $\mathrm{Cu}(+0.34 \mathrm{~V}$ vs standard hydrogen electrode (SHE)) is less than that of the metal ions in solution, different metal NPs such as $\mathrm{Ag}(+0.78 \mathrm{~V}$ vs SHE) and $\mathrm{Au}(+1.5 \mathrm{~V}$ vs SHE) can also be effectively deposited onto graphene-defect sites as well as Pd (+0.83 V vs SHE). To clarify this point, we also tested the galvanic displacement reaction with an $\mathrm{AgNO}_{3}$ solution and observed the selective depositions of $\mathrm{Ag}$ onto graphene-defect sites, as shown in Supplementary Figure S6. Thus, various metal NPs can be decorated onto the graphene-defect sites through the use of the galvanic displacement reaction and further used for the functionalization of graphene.

The effect of the wet-chemistry-based galvanic displacement reaction on CVD-grown graphene was examined by a comparison of the electrical sheet resistance changes. The sheet resistance of CVD-grown SLG is typically greater than $1 \mathrm{k} \Omega \mathrm{sq} .{ }^{-1}$, which is much higher than the theoretically calculated value $^{3}$ (approximately $30 \Omega$ sq. $^{-1}$ for pristine graphene) because of inherent defects, such as GBs, shallow lines because of $\mathrm{Cu}$ fatigue cracks below SLG and wrinkles. ${ }^{14,38}$ Although these graphene defects can be minimized by controlling the CVD growth conditions, GBs cannot be eliminated because of the polycrystalline nature of CVD-grown graphene. The increased sheet resistance of the CVD-grown graphene compared with the ideal value is because of the delayed charge transport on the defective sites. ${ }^{39}$ If the defective sites of the graphene are decorated with metallic NPs, then the electrical conduction can be improved because of the increased conduction path and the increased carrier density through chemical doping by metallic NPs. Several studies have demonstrated that the sheet resistance of graphene can be improved by decorating graphene with metal nanowires and metal nanoparticles. ${ }^{14-16}$ Therefore, the selective deposition of Pd NPs via the galvanic displacement reaction can be a more effective method for the decoration of graphene defects because the galvanic reaction is more cost-effective than others methods, such as vapor-phase treatment and the bridging method with metal nanowires. ${ }^{14-17}$ The optical transmittance and sheet resistance of the SLG samples were measured after galvanic displacement reactions for various times and at various concentrations to investigate the effects of selective Pd deposition on SLG defects. The pristine graphene sample had an optical transmittance $(T)$ of approximately $97 \%$ at a wavelength of $550 \mathrm{~nm}$, which is comparable to previously reported results. ${ }^{15}$ After the $\mathrm{Pd}$ was deposited onto SLG for $10 \mathrm{~min}$ in a $0.1-\mathrm{mm} \mathrm{PdCl}_{2}$ solution, $T$ slightly decreased to $91.3 \%$ when measured at $500 \mathrm{~nm}$ because of light blockage by Pd NPs (Figure 6a). We also measured the sheet resistance of the Pd NP-deposited graphene and corresponding optical transmittance, as shown in Figure 6b. The sheet resistance of pristine graphene was approximately $1 \mathrm{k} \Omega \mathrm{sq} .^{-1}$ under ambient conditions. When the $\mathrm{PdCl}_{2}$ concentration and galvanic displacement reaction time increased, the sheet resistance rapidly decreased and reached $173 \Omega$ sq. ${ }^{-1}$ at $T=91.3 \%$, which is a huge decrease from the initial sheet resistance (a decrease of approximately 83\%). This decrease compares with the similar decrease in sheet resistance to approximately $125 \Omega \mathrm{sq}^{-1}$, with a superior transmittance of $\mathrm{T}=97.4 \%$ previously reported via $\mathrm{HNO}_{3}$ doping. ${ }^{14}$ This value was obtained after 
a

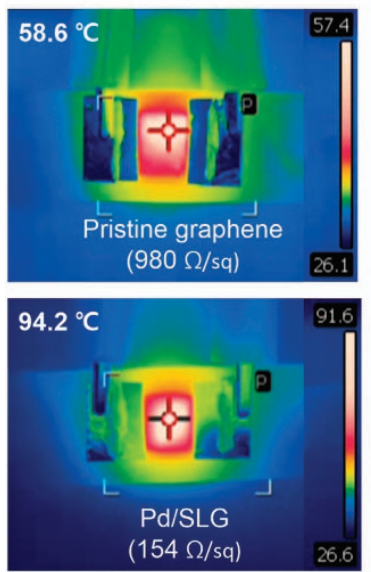

C

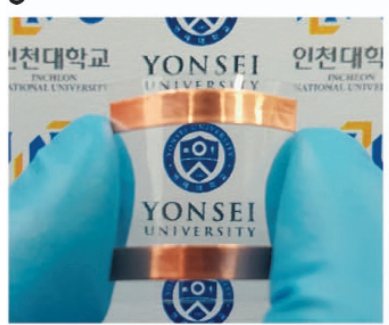

b

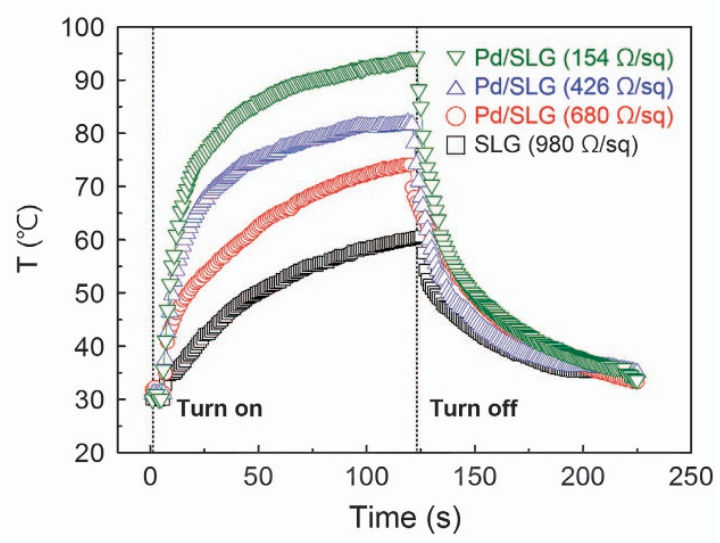

d

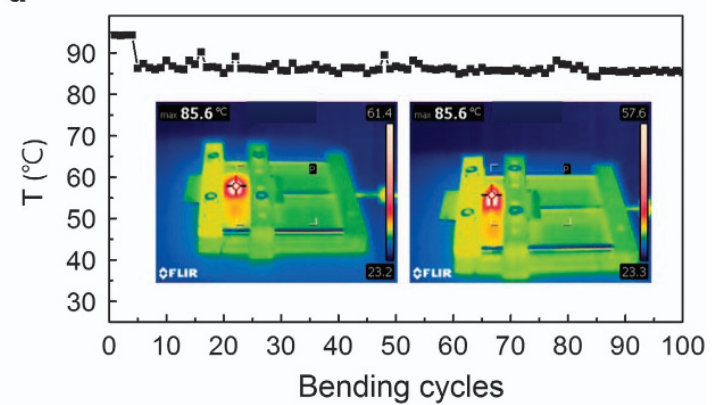

Figure 7 (a) Infrared thermal images of pristine graphene and Pd-decorated graphene heaters $(20 \mathrm{~mm} \times 20 \mathrm{~mm}$ ) under an applied 12-V input voltage. Cursors indicate the maximum temperatures. (b) Time-dependent temperature profiles of pristine graphene and Pd-decorated graphene heaters with respect to the sheet resistances. (c) A photograph of the Pd-decorated graphene heater, showing its mechanical flexibility and optical transparency. (d) Temperature change of a Pd-decorated graphene heater under bending testing. The inset infrared thermal images show the bending process.

the galvanic displacement reaction in the $0.1-\mathrm{mM} \mathrm{PdCl}_{2}$ solution for $10 \mathrm{~min}$. Under these conditions, the Pd NPs were completely connected and formed nanowire-like structures without gaps between Pd NPs (Supplementary Figures S1c-f), thereby efficiently providing electrical conduction paths. Alternatively, the surface coverage of Pd NPs on SLG increased with decreasing sheet resistance, which demonstrates that Pd NPs on SLG effectively provide extra conduction paths. The optical transmittance decreased as the sheet resistance decreased because of increased surface coverage; however, the optical transmittance was still sufficiently high to allow the material to be used as a transparent electrode.

Micro-Raman spectroscopy was used to investigate the doping effects of Pd NPs on SLG. The Raman spectrum of pristine graphene shows the common $\mathrm{G}$ and $2 \mathrm{D}$ peaks of graphene, which are located at approximately 1580 and $2680 \mathrm{~cm}^{-1}$, respectively, as shown in Figures $6 \mathrm{c}$,d. When both the galvanic displacement reaction time and the solution concentration were increased, both the $G$ and 2D peaks gradually blueshifted and showed a substantial change from 1580 to $1595 \mathrm{~cm}^{-1}$ and 2680 to $2688 \mathrm{~cm}^{-1}$, respectively, for the SLG reacted in the $0.1-\mathrm{mM} \mathrm{PdCl}_{2}$ solution for $10 \mathrm{~min}$. The simultaneous blueshifts in the $\mathrm{G}$ and $2 \mathrm{D}$ peaks indicate hole doping of graphene by Pd NPs. ${ }^{27,28}$ This result indicates that the selectively deposited Pd NPs on graphene-defect sites contribute to electrical conduction by doping and to the formation of additional conduction paths. However, it was not easy to determine which effect was dominant with the current experimental setup.
To demonstrate the potential of defect-healed graphene as a transparent electrode, we fabricated Pd-decorated graphene heaters with varying coverage of Pd NPs by using the galvanic displacement reaction and compared the results with the heating performance of a pristine graphene heater. To decrease heat loss, the PMMA-supporting layers above Pd-decorated graphene and pristine graphene were left intact. A direct current power source was connected to the $\mathrm{Cu}$ contacts below the graphene layer, and its output voltage was kept constant $(12 \mathrm{~V})$. The temperature distribution of heaters was simultaneously monitored using an infrared thermal imaging camera. Figure 7a shows the infrared thermal images of a pristine graphene heater and the Pd-decorated graphene heater. Both the pristine graphene and Pd-decorated graphene heaters show uniform temperature distributions, whereas they show different maximum temperatures at steady-state because of the difference in their sheet resistances. Figure $7 \mathrm{~b}$ shows the time-dependent temperature profiles of the pristine graphene and the Pd-decorated graphene heater with respect to the sheet resistance of the film. Notably, the maximum temperature at steady-state increases as the sheet resistance of the heater decreases under the same input voltage $(12 \mathrm{~V})$, which is in accord with previously reported results. ${ }^{40}$ The power consumption values were theoretically calculated based on the experimental values for pristine SLG and Pd-decorated graphene with diverse sheet resistances (Supplementary Table S1). The Pd-decorated graphene heater with $154 \Omega$ sq. $^{-1}$, with a steady-state temperature as high as $94.4^{\circ} \mathrm{C}$ (Supplementary Video 1) showed better heating performance than 
the pristine graphene heater, which showed a maximum steady-state temperature of $60.6{ }^{\circ} \mathrm{C}$ (Supplementary Video 2). These results indicate that our Pd-decorated graphene heaters effectively operated as transparent heaters with sufficiently high electrical conductivities.

Given the advantage of graphene in flexibility, as shown in Figure $7 c$, we tested the mechanical stability and reliability of the Pd-decorated graphene heater $\left(20 \times 20 \mathrm{~mm}^{2}\right)$ by evaluating the variation in temperature under continuous bending cycles (a curvature of $10 \mathrm{~mm}$ ). Figure $7 \mathrm{~d}$ shows the temperature changes of the Pd-decorated graphene heater with $154 \Omega$ sq. ${ }^{-1}$ during bending cycle tests, and the inset in Figure $7 \mathrm{~d}$ shows infrared thermal images before and after bending with a curvature of $10 \mathrm{~mm}$. Notably, the maximum steady-state temperature was maintained for a short moment in the early stage of the bending test, and the maximum steady-state temperature decreased to $85.6{ }^{\circ} \mathrm{C}$ (a decrease of approximately 9\%); this temperature was maintained under continued bending testing. The decrease of the maximum steady-state temperature in the early bending state is attributed to the unavoidable detachment of Pd NPs from the graphene surface and to increased contact resistance because of bending. Despite a slight decrease in the maximum steady-state temperature in the initial bending stage, the stabilized steady-state temperature was not changed under repeated bending tests. In addition, the theoretically calculated power consumption values for both flat and bending test samples were almost the same, indicating that the heater properties and performance are not degraded by bending (Supplementary Table S2). Consequently, the result in the bending cycle test indicates that our Pd-decorated graphene heater can even be operated under mechanical strain without substantial degradation in its heating performance.

\section{CONCLUSION}

We demonstrated an effective decoration method for inherent atomic defects on CVD-grown graphene via a wet-chemistry-based galvanic displacement reaction. $\mathrm{C}_{\mathrm{s}}$-corrected STEM, FE-SEM and AFM analyses confirmed that the simple redox reaction between $\mathrm{Cu}$ and $\mathrm{Pd}^{2+}$ ions predominantly occurred on the graphene-defect sites because of increased chemical reactivity on the graphene imperfection sites, such as GBs, $\mathrm{Cu}$ fatigue cracks, wrinkles and multiple layers. All the defects on graphene were spontaneously healed after the galvanic displacement reaction using a $\mathrm{PdCl}_{2}$ solution with a concentration higher than or equal to 0.1 but less than or equal to $0.5 \mathrm{~mm}$. Each defect was identified by distinguishing different Pd deposition shapes on the SLG surface. More importantly, the defect-healed graphene showed a noticeable improvement in electrical sheet resistance and was successfully operated as a transparent heater by yielding better heating performance compared with a heater fabricated using pristine graphene. This result indicates that graphene can be used as an effective transparent conducting electrode, which further increases opportunities for the future development of electronic devices in a broad range of applications. Our approach can also be expanded to other large-scale two-dimensional materials, such as boron nitride and metal transition dichalcogenides.

\section{CONFLICT OF INTEREST}

The authors declare no conflict of interest.

\section{ACKNOWLEDGEMENTS}

This work was supported by the Priority Research Centers Program (Grant No. 2012-0006689) through the National Research Foundation (NRF) of Korea funded by the Ministry of Education, Science and Technology (MEST) and the Mid-career Researcher Program through an NRF grant funded by the MEST
(Grant No. 2014R1A2A2A09053061). In addition, this research was supported by the Basic Science Research Program through the National Research Foundation of Korea (NRF) funded by the Ministry of Education (2014R1A1A2059845). HL and JYP acknowledge the support by IBS-R004-G4. We thank Tanaka Kikinzoku Kogyo K.K. for comments on the usage of the $\mathrm{PdCl}_{2}$ solution. This work was partially supported by the Yonsei University Future-leading Research Initiative.

1 Wu, Y., Lin, Y.-M., Bol, A. A., Jenkins, K. A., Xia, F., Farmer, D. B., Zhu, Y. \& Avouris, P. High-frequency, scaled graphene transistors on diamond-like carbon. Nature 472, 74-78 (2011).

2 Zhang, F., Zhang, T., Yang, X., Zhange, L., Leng, K., Huang, Y. \& Chen, Y. A high-performance supercapacitor-battery hybrid energy storage device based on graphene-enhanced electrode materials with ultrahigh energy density. Energy Environ. Sci. 6, 1623-1632 (2013).

3 Bonaccorso, F., Sun, Z., Hasan, T. \& Ferrari, A. C. Graphene photonics and optoelectronics. Nat. Photonics 4, 611-622 (2010).

4 Chae, S. J., Güneş, F., Kim, K. K., Kim, E. S., Han, G. H., Kim, S. M., Shin, J. H., Yoon, S. M., Choi, J. Y., Park, M. H., Yang, C. W., Pribat, D. \& Lee, Y. H. Synthesis of large-area graphene layers on poly-nickel substrate by chemical vapor deposition: wrinkle formation. Adv. Mater. 21, 2328-2333 (2009).

5 Pereira, V. M., Guinea, F., Lopes dos Santos, J. M. B., Peres, N. M. R. \& Castro Neto, A. H. Disorder induced localized states in graphene. Phys. Rev. Lett. 96, 036801 (2006).

6 Kim, K., Lee, Z., Regan, W., Kisielowski, C., Crommie, M. F. \& Zettl, A. Grain boundary mapping in polycrystalline graphene. ACS Nano 5, 2142-2146 (2011).

7 Huang, P. Y., Ruiz-Vargas, C. S., van der Zande, A. M., Whitney, W. S., Levendorf, M. P., Kevek, J. W., Garg, S., Alden, J. S., Hustedt, C. J., Zhu, Y., Park, J., McEuen, P. L. \& Muller, D. A. Grains and grain boundaries in single-layer graphene atomic patchwork quilts. Nature 469, 389-392 (2011).

8 Kwon, S., Chung, H. J., Seo, S. \& Park, J. Y. Domain structures of single layer graphene imaged with conductive probe atomic force microscopy. Surf. Interface Anal. 44, 768-771 (2012).

9 Zhu, W., Low, T., Perebeinos, V., Bol, A. A., Zhu, Y., Yan, H., Tersoff, J. \& Avouris, P. Structure and electronic transport in graphene wrinkles. Nano Lett. 12, 3431-3436 (2012).

10 Meyer, J. C., Geim, A. K., Katsnelson, M. I., Novoselov, K. S., Booth, T. J. \& Roth, S. The structure of suspended graphene sheets. Nature 446, 60-63 (2007).

11 Ovid'ko, I. A. \& Sheinerman, A. G. Cracks at disclinated grain boundaries in graphene. J. Phys. D Appl. Phys. 46, 345305 (2013).

12 Li, X., Magnuson, C. W., Venugopal, A., An, J., Suk, J. W., Han, B., Borysiak, M., Cai, W., Velamakanni, A., Zhu, Y., Fu, L., Vogel, E. M., Voelkl, E., Colombo, L. \& Ruoff, R. S. Graphene films with large domain size by a two-step chemical vapor deposition process. Nano Lett. 10, 4328-4334 (2010).

13 Yu, Q., Jauregui, L. A., Wu, W., Colby, R., Tian, J., Su, Z., Cao, H., Liu, Z., Pandey, D., Wei, D., Chung, T. F., Peng, P., Guisinger, N. P. Stach, E. A., Bao, J., Pei, S. S. \& Chen, Y. P. Control and characterization of individual grains and grain boundaries in graphene grown by chemical vapour deposition. Nat. Mater. 10, 443-449 (2011).

14 Bae, S., Kim, H., Lee, Y., Xu, X., Park, J. S., Zheng, Y., Balakrishnan, J., Lei, T., Kim, H. R., Song, Y. I., Kim, Y. J., Kim, K. S., Ozyilmaz, B., Ahn, J. H., Hong, B. H. \& lijima, S. Roll-to-roll production of 30-inch graphene films for transparent electrodes. Nat. Nanotechnol. 5, 574-578 (2010).

15 Kim, K., Lee, H.-B.-R., Johnson, R. W., Tanskanen, J. T., Liu, N., Kim, M.-G., Pang, C., Ahn, C., Bent, S. F. \& Bao, Z. Selective metal deposition at graphene line defects by atomic layer deposition. Nat. Commun. 5, 4781 (2014).

16 Van Lam, D., Kim, S.-M., Cho, Y., Kim, J.-H., Lee, H.-J., Yang, J.-M. \& Lee, S. M. Healing defective CVD-graphene through vapor phase treatment. Nanoscale 6, 5639-5644 (2014).

17 Oh, I.-K., Tanskanen, J., Jung, H., Kim, K., Lee, M. J., Lee, Z., Lee, S. K., Ahn, J. H., Lee, C. W., Kim, K., Kim, H. \& Lee, H.-B.-R. Nucleation and growth of the $\mathrm{HfO}_{2}$ dielectric layer for graphene-based devices. Chem. Mater. 27, 5868-5877 (2015).

18 Ho, P.-H., Liou, Y.-T., Chuang, C.-H., Lin, S.-W., Tseng, C.-Y., Wang, D.-Y., Chen, C. C., Hung, W. Y., Wen, C. Y. \& Chen, C. W. Self-crack-filled graphene films by metallic nanoparticles for high-performance graphene heterojunction solar cells. Adv. Mater. 27, 1724-1729 (2015)

19 Son, J.-H., Baeck, S.-J., Park, M.-H., Lee, J.-B., Yang, C.-W., Song, J.-K., Zin, W. C. \& Ahn, J. H. Detection of graphene domains and defects using liquid crystals. Nat. Commun. 5, 3484 (2014).

20 Hong, J., Lee, S., Seo, J., Pyo, S., Kim, J. \& Lee, T. A highly sensitive hydrogen sensor with gas selectivity using a PMMA membrane-coated Pd nanoparticle/single-Layer graphene hybrid. ACS Appl. Mater. Interfaces 7, 3554-3561 (2015).

21 Regan, W., Alem, N., Alemán, B., Geng, B., Girit, Ç., Maserati, L., Wang, F., Crommie, M. \& Zettl, A. A direct transfer of layer-area graphene. Appl. Phys. Lett. 96, 113102 (2010).

22 Lee, H., Lee, H.-B.-R., Kwon, S., Salmeron, M. \& Park, J. Y. Internal and external atomic steps in graphite exhibit dramatically different physical and chemical properties. ACS Nano 9, 3814-3819 (2015).

23 Liu, N., Pan, Z., Fu, L., Zhang, C., Dai, B. \& Liu, Z. The origin of wrinkles on transferred graphene. Nano Res. 4, 996-1004 (2011). 
24 Duan, W. H., Gong, K. \& Wang, Q. Controlling the formation of wrinkles in a single layer graphene sheet subjected to in-plane shear. Carbon 49, 3107-3112 (2011).

25 Yu, S. U., Park, B., Cho, Y., Hyun, S., Kim, J. K. \& Kim, K. S. Simultaneous visualization of graphene grain boundaries and wrinkles with structural information by gold deposition. ACS Nano 8, 8662-8668 (2014).

26 Duong, D. L., Han, G. H., Lee, S. M., Gunes, F., Kim, E. S., Kim, S. T., Kim, H., Ta, Q. H., So, K. P., Yoon, S. J., Chae, S. J., Jo, Y. W., Park, M. H., Chae, S. H., Lim, S. C., Choi, J. Y. \& Lee, Y. H. Probing graphene grain boundaries with optical microscopy. Nature 490, 235-239 (2012).

27 Casiraghi, C., Pisana, S., Novoselov, K. S., Geim, A. K. \& Ferrari, A. C. Raman fingerprint of charged impurities in graphene. Appl. Phys. Lett. 91, 233108 (2007).

28 Zhang, L., Yu, J., Yang, M., Xie, Q., Peng, H. \& Liu, Z. Janus graphene from asymmetric two-dimensional chemistry. Nat. Commun. 4, 1443 (2013).

29 Li, Z., Kinloch, I. A., Young, R. J., Novoselov, K. S., Anagnostopoulos, G., Parthenios, J., Galiotis, C., Papagelis, K., Lu, C. Y. \& Britnell, L. Deformation of wrinkled graphene. ACS Nano 9, 3917-3925 (2015).

30 Durgun, E., Dag, S., Bagci, V. M. K., Gülseren, O., Yildirim, T. \& Ciraci, S. Systematic study of adsorption of single atoms on a carbon nanotube. Phys. Rev. B 67, 201401 (2003).

31 Lee, H.-B.-R., Baeck, S. H., Jaramillo, T. F. \& Bent, S. F. Growth of Pt nanowires by atomic layer deposition on highly ordered pyrolytic graphite. Nano Lett. 13 457-463 (2013).

32 Brown, L., Hovden, R., Huang, P., Wojcik, M., Muller, D. A. \& Park, J. Twinning and twisting of tri- and nilayer graphene. Nano Lett. 12, 1609-1615 (2012).

33 Gan, L., Zhang, H., Wu, R., Zhang, Q., Ou, X., Ding, Y., Sheng, P. \& Luo, Z. Grain size control in the fabrication of large single-crystal bilayer graphene structures. Nanoscale 7, 2391-2399 (2015)

34 Wu, P., Zhai, X., Li, Z. \& Yang, J. Bilayer graphene growth via a penetration mechanism. J. Phys. Chem. C 118, 6201-6206 (2014)
35 Qu, L. \& Dai, L. Substrate-enhanced electroless deposition of metal nanoparticles on carbon nanotubes. J. Am. Chem. Soc. 127, 10806-10807 (2005).

36 Shen, A., Zou, Y., Wang, Q., Dryfe, R. A. W., Huang, X., Dou, S., Dai, L. \& Wang, S. Oxygen reduction reaction in a droplet on graphite: direct evidence that the edge Is more active than the basal plane. Angew. Chem. Int. Ed. 53, 10804-10808 (2014).

37 Li, Z., Zhang, P., Wang, K., Xu, Z., Wei, J., Fan, L., Wu, D. \& Zhu, H. Graphene buffered galvanic synthesis of graphene-metal hybrids. J. Mater. Chem. 21, 13241-13246 (2011).

38 Suk, J. W., Kitt, A., Magnuson, C. W., Hao, Y., Ahmed, S., An, J., Swan, A. K., Goldberg, B. B. \& Ruoff, R. S. Transfer of CVD-grown monolayer graphene onto arbitrary substrates. ACS Nano 5, 6916-6924 (2011).

39 Yazyev, O. V. \& Louie, S. G. Electronic transport in polycrystalline graphene. Nat. Mater. 9, 806-809 (2010)

40 Kang, J., Kim, H., Kim, K. S., Lee, S. K., Bae, S., Ahn, J. H., Kim, Y. J., Choi, J. B. \& Hong, B. H. High-performance graphene-based transparent flexible heaters. Nano Lett. 11, 5154-5158 (2011).

(c) (i) This work is licensed under a Creative Commons Attribution 4.0 International License. The images or other third party material in this article are included in the article's Creative Commons license, unless indicated otherwise in the credit line; if the material is not included under the Creative Commons license, users will need to obtain permission from the license holder to reproduce the material. To view a copy of this license, visit http:// creativecommons.org/licenses/by/4.0/

Supplementary Information accompanies the paper on the NPG Asia Materials website (http://www.nature.com/am) 